ANZIAM JOURNAL

ACCEPTED 20 JANUARY, 2016

ISSN 1445-8735

PUBLISHER: CAMBRIDGE UNIVERSITY PRESS, UK

\title{
UNSTEADY TWO-LAYERED BLOOD FLOW THROUGH A W-SHAPE STENOSED ARTERY USING THE GENERALIZED OLDROYD-B FLUID MODEL
}

\author{
Nasir Ali ${ }^{\mathrm{a}, 1}$, Akbar Zaman $^{\mathrm{a}}$, O. Anwar Bég ${ }^{\mathrm{b}}$ and M. Sajid ${ }^{\mathrm{c}}$ \\ ${ }^{a}$ Department of Mathematics and Statistics, International Islamic University, \\ Islamabad, 44000, Pakistan. \\ ${ }^{b}$ Spray Research Group, Petroleum and Gas Engineering Division, School of \\ Computing, Science and Engineering (CSE), University of Salford, M5 4WT, UK. \\ ${ }^{c}$ Theoretical Physics Division, PINSTECH, P.O. Nilore, Islamabad, 44000, Pakistan.
}

\begin{abstract}
A theoretical study of unsteady two-layered blood flow through a stenosed artery is presented in this article. The geometry of rigid stenosed artery is assumed to be $w$-shaped. The flow regime is assumed to be laminar, unsteady and uni-directional. The characteristics of blood are modeled by the generalized Oldroyd-B non-Newtonian fluid model in the core region and a Newtonian fluid in the periphery region. The governing partial differential are derived for each region by using mass and momentum conservation equations. In order to facilitate numerical solutions, the derived differential equations are non-dimensionalized. A well-tested explicit finite difference scheme (FDM) which is forward in time and central in space is employed for the solution of nonlinear initial-boundary value problem corresponding to each region. Validation of the FDM computations is achieved with a variational finite element method (FEM) algorithm. The influence of the emerging geometric and rheological parameters on axial velocity, resistance impedance and wall shear stress are displayed graphically. The instantaneous patterns of streamlines are also presented to illustrate the global behavior of blood flow. The simulations are relevant to hemodynamics of small blood vessels and capillary transport wherein rheological effects are dominant.
\end{abstract}

Keywords: Unsteady two layer flow; Generalized Oldroyd-B fluid; Finite difference method; Hemodynamics; Stenosis; Finite element method; Streamline visualization.

${ }^{1}$ Corresponding author: Tel :-+92519019756 (e-mail: nasirali_qau@yahoo.com). 


\section{INTRODUCTION}

The composition of blood is known to be that of a multi-component mixture of aqueous plasma, red and white blood cells RBCs, WBCs and a variety of lipoproteins [1]. The fact that rheological characteristics of blood influence many of the pathological conditions observed in the circulatory system has simulated research in two distinct directions with regard to the constitutive modeling of blood. The first approach is more general and is founded on the micro-continuum theories and emphasizes the use of additional balance laws to characterize the rheology of blood. The second approach simulates blood as a suspension and implements the use of techniques of macroscopic non-Newtonian fluid mechanics. In view of the former approach, the rheology of blood can be successfully characterized by the constitutive equations of micropolar, dipolar, couple stress and micromorphic fluid models. The later approach allows a variety of of constitutive equations to be deployed to represent the shear stress-strain behavior of blood depending on different non-Newtonian characteristics. It is also well established that blood exhibits non-linear properties due to presence of red blood cells (RBCs) which form approximately $45 \%$ of volume of normal human blood, under certain flow conditions. Typical non-Newtonian characteristics exhibited by blood are shear-thinning, thixotropy, viscoelasticity and yield stress. Time-independent non-Newtonian models are commonly used in the literature to capture the shear thinning property of blood. These include the power-law model [2], Carreau model [3], Casson model [4], Walburn-Schneck model [5], Cross model [6] and Weaver model [7] to name only a few. Thixotropic and yield stress behavior of blood is described quite accurately by time-dependent non-Newtonian models. Some well-known time independent thixotropic models used in the literature on blood flow are the Huang model [8], Weltman model [9], TiuBoger [9] and Rosen model [10]. As emphasized by Thurston [11] the viscoelastic behavior of blood becomes prominent at low shear rates due to aggregation of RBCs. Therefore under pulsatile flow conditions the blood may be modeled as a viscoelastic fluid. Popular viscoelastic fluid models in hemodynamics which are adopted to characterize blood rheology include the Oldroyd-B fluid model, Yeleswarapu model [12], generalized Maxwell model [13] and generalized Oldroyd-B model [14].

Significant experimental evidence also exists demonstrating that rheological properties of blood may change with variations in the diameter of the vessel. For instance, it has been observed that the measured apparent viscosity of blood decreases with reducing diameter of the blood vessel. This phenomenon is known as the Fahreaus-Lindqvist (F-L) effect [15]. This effect may be due to migration of RBCs away from the wall resulting in formation of a cell-depleted layer near the wall. Many researchers have modelled blood as a two-layered fluid to capture the FL effect. The peripheral/cell-depleted plasma layer is modeled as a Newtonian fluid while the core region (mixture of RBCs and plasma) is modeled as a non-Newtonian fluid. Following this approach Majhi and Usha [16] modeled the core region using the 
thermodynamically robust constitutive equation of third grade fluids to capture the FL effect. Subsequently Majhi and Nair [17] extended their previous analysis to include the effect of pulsatile conditions and body acceleration. Massoudi and Phuoc [18] analyzed the blood in the core region as a modified second grade fluid to study pulsatile flow of blood in a normal artery. Recently Sajid et al. [19] analyzed the pulsatile flow of blood utilizing the constitutive equation of Oldroyd-B fluid to model the core region, and therein also elaborated on the effects of relaxation and retardation constant on various hemodynamic characteristics.

In above mentioned studies the results are reported for a normal artery. The practical significance of these results is limited as they do not elucidate the fluid dynamics characteristics of blood in diseased/stenotic arteries. This has motivated some researchers to conduct similar investigations for stenotic arteries. Mention may be made of the work of Ikbal et al. [20] which considers the unsteady two-layered blood flow through a flexible artery under stenotic conditions using Eringen's elegant micropolar fluid model. Sankar and Lee [21] have analyzed steady flow of blood through a catheterized artery via a two-fluid model with the core region approximated as a Casson fluid and the plasma in the peripheral region as a Newtonian fluid. Motivated by extending and improving above studies, we propose, in this paper, to examine theoretically the unsteady two-layered pulsatile flow of blood through a stenosed artery. The viscoelastic and shear-thinning effects of blood are both simultaneously important in the pulsatile flow under consideration. In this context, a model which is capable of concurrently predicting the shearthinning and visco-elastic effects may be more appropriate. The generalized Oldroyd-B model [22-23] is quite suitable for the present flow situation as it is able to capture shear thinning, viscoelastic effects and based on thermodynamic considerations, permits the relaxation time to be a constant. It includes a shearrate dependent viscosity and has proved effective in numerous hemodynamic (and indeed other) scenarios. The Oldroyd-B model has earlier been employed to investigate pulsatile flow of blood in a tube of infinite length and circular cross-section [24]. The results in ref. [25] highlight the combined effects of the viscoelasticity and of shear dependent viscosity. The present study also generalizes the results of ref. [24] for an overlapping stenosed artery. Moreover, it includes the results of the conventional Oldroyd-B fluid, Maxwell fluid, Carreau fluid and Newtonian (Navier-Stokes) fluids as special cases. The coupled constitutive and motion equations are solved numerically using a stable, convergent, explicit finite difference method (FDM) which is forward in time and central in space. The results of velocity obtained via this method are first validated with a variational finite element method and then the flow characteristics of blood for various models (which include Oldroyd-B, Maxwell, Carreau and Newtonian models) are compared and discussed in detail. The present article addresses in summary, the unsteady two layer blood flow through a $w$-shape stenosed artery with a generalized Oldroyd-B rheological fluid 
model. The geometry of the overlapping stenosis is carefully defined. Viscous flow characteristics of blood in a stenosed artery for Carreau, Olroyd-B model and Maxwell models are compared. The paper is structured as follows: The flow geometry is illustrated in Section 2. The problem is modeled with appropriate assumptions in section 3. Section 4 presents the detail of numerical procedure for solution of the developed equations. The obtained results are discussed in section 5. The main conclusions of the present study are summarized in section 6.

\section{PROBLEM DESCRIPTION}

A homogenous incompressible two-layered fluid (blood) is assumed to be flowing through an overlapping stenosed artery of length $L$. A cylindrical coordinate system $(r, \theta, z)$, is employed for the current analysis. The mathematical equation describing the geometry of the stenosis and interface is given as:

$$
\left(R, R_{1}\right)(z)=\left\{\begin{array}{cc}
\left(a, a^{*}\right)-\frac{128}{5} \frac{\left(\delta^{*}, \tau^{*}\right)}{l_{0}^{4}}\left(\frac{11}{32} l_{0}^{3}(z-d)-\frac{47}{48} l_{0}^{2}(z-d)^{2}+l_{0}(z-d)^{3}-\frac{1}{3}(z-d)^{4}\right), d \leq z \leq d+\frac{3}{2} l_{0}, \\
\left(a, a^{*}\right) & \text { otherwise. }
\end{array}\right.
$$

In the above expression $R(z)$ denotes the radius of the arterial segment with stenosis, $\delta^{*}$ is the maximum height of the stenosis, $a$ is the constant radius of the artery in the non-stenotic region, $a^{*}$ radius of a core region, $l_{0}$ is the length of the stenosis, $d$ is the length of the pre-stenotic region, $\tau^{*}$ is the bulging of interface and $R_{1}(z)$ denotes the radius of the central core layer. A schematic diagram of the non-tapered artery is shown in Fig. 1.

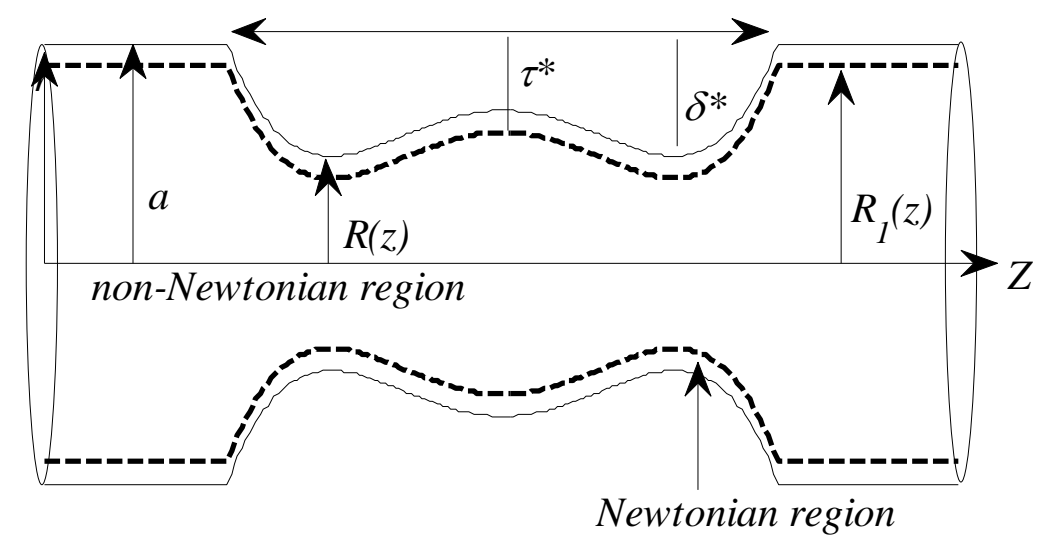

Fig. 1: Geometry of the overlapping stenotic artery 
Note that $\left(\delta^{*}, \tau^{*}\right)$ denote the critical heights of the two-layered stenosis appearing at two specific locations i.e.,

$$
z=d+\frac{9 l_{0}}{50} \text {, and } z=d+\frac{41 l_{0}}{50}
$$

\section{HEMODYNAMIC MODEL}

The equations governing the blood flow are:

$$
\begin{aligned}
& U_{, i}^{i}=0, \\
& \rho a^{i}=T_{, j}^{i j},
\end{aligned}
$$

where $V^{i}$ are the velocity components, $\rho$ is the fluid density, $T^{i j}$ are the components of Cauchy stress tensor, "," denotes the covariant derivative and $a^{i}$ are the components of the acceleration vector given as:

$$
a^{i}=\frac{\partial U^{i}}{\partial t}+U^{r} U_{, r}^{i}
$$

The constitutive law for incompressible fluid takes the form:

$$
T^{i j}=-p \delta^{i j}+S^{i j}
$$

in which $p$ is the pressure, $\delta^{i j}$ is the identity tensor and $S^{i j}$ is the extra stress tensor.

We have delineated the arterial domain into two distinct regions: $0 \leq r \leq R_{1}$ as (a core region) and $R_{1} \leq r \leq R$ (a periphery region). For the present case, the rheology of blood in the core is characterized by the constitutive equation of a generalized Oldroyd-B fluid while in the periphery region it is assumed to satisfy the well-known Newtonian constitutive law. Thus the equation for $S^{i j}$ in the core region is [26],

$$
S^{i j}+\lambda_{1} \frac{D S^{i j}}{D t}=\left[\mu(\Pi)+\mu_{0} \lambda_{2} \frac{D}{D t}\right] A_{1}^{i j}, \quad 0 \leq r \leq R_{1},
$$

where

$$
\mu(\Pi)=\left(\mu_{\infty}+\left(\mu_{0}-\mu_{\infty}\right)\left[1+\Gamma^{2} \Pi^{2}\right]^{(n-1) / 2}\right)
$$

The equation of $S^{i j}$ in the periphery region is defined as:

$$
S^{i j}=\mu_{1} A_{1}^{i j}, \quad R_{1} \leq r \leq R .
$$


In Eqns. (7) and (9), $\mu_{0}$ and $\mu_{\infty}$ are zero-shear-rate and infinite-shear-rate viscosities, respectively, $\Gamma$ is the time constant, $n$ is the power law index, $\lambda_{1}$ and $\lambda_{2}$ are relaxation and retardation times, respectively and $A_{1}^{i j}$ are the components of the first Rivlin-Ericksen tensor given by:

$$
A_{1}^{i j}=U_{, j}^{i}+U_{, i}^{j}
$$

The second invariant of first Rivlin-Ericksen tensor $\Pi$ is defined as:

$$
\Pi=\sqrt{\frac{1}{2}\left(A_{1}^{p k} A_{1}^{k p}\right)}
$$

Under mild stenotic conditions, the flow in the artery can be treated as uni-directional and unsteady. Let $u_{1}, u_{2}$ denote non-vanishing axial velocity components in core and peripheral region, respectively. From a momentum balance (4), the axial velocity component in the peripheral region satisfies:

$$
\rho_{2} \frac{\partial u_{2}}{\partial t}=-\frac{\partial p}{\partial z}+\frac{1}{r} \frac{\partial}{\partial r}\left(r S^{r z}\right)
$$

where $S^{r z}$ is given through

$$
S^{r z}=\mu_{1} \frac{\partial u_{2}}{\partial r}, \quad R_{1} \leq r \leq R
$$

and $\rho_{2}$ is the fluid density in the peripheral region.

Similarly, the axial velocity component in the core region satisfies:

$$
\rho_{1} \frac{\partial u_{1}}{\partial t}=-\frac{\partial p}{\partial z}+\frac{1}{r} \frac{\partial}{\partial r}\left(r S^{r z}\right)
$$

where $\rho_{1}$ is the fluid density in the core region. In view of (7), $S^{r z}$ in the core region satisfies the following equation [27],

$$
\left(1+\lambda_{1} \frac{\partial}{\partial t}\right) S_{r z}=\left(\left[\mu_{\infty}+\left(\mu_{0}-\mu_{\infty}\right)\left\{1+\Gamma^{2}\left|\frac{\partial u_{1}^{2}}{\partial r}\right|\right\}^{n-1 / 2}\right]+\mu_{0} \lambda_{2} \frac{\partial}{\partial t}\right) \frac{\partial u_{1}}{\partial r}, \quad 0 \leq r \leq R_{1} .
$$

Since the flow is initiated by the pulsatile pressure gradient, therefore following [28], we write: 


$$
-\frac{\partial p}{\partial z}=A_{0}\left(1+\frac{A_{1}}{A_{0}} \cos \omega_{p} t\right)
$$

Here $A_{0}$ is the systolic and $A_{1}$ is the diastolic component of the pressure gradient, $\omega_{p}=2 \pi f_{p}$ is the circular frequency and $f_{p}$ is the pulse rate frequency. Eliminating $S_{r z}$ between Eqs. (12) and (14), we get the equation for the velocity components in the core region as follows:

$$
\begin{aligned}
& \rho_{1}\left(\frac{\partial u_{1}}{\partial t}+\lambda_{1} \frac{\partial^{2} u_{1}}{\partial t^{2}}\right)=A_{0}\left(1+\frac{A_{1}}{A_{0}} \cos \left(\omega_{p} t\right)-\lambda_{1} \frac{A_{1}}{A_{0}} \omega_{p} \sin \left(\omega_{p} t\right)\right)+\frac{\mu_{0}}{r}\left[\left\{\frac{\mu_{\infty}}{\mu_{0}}+\left(1-\frac{\mu_{\infty}}{\mu_{0}}\right)\left\{1+\Gamma^{2}\left|\frac{\partial u_{1}{ }^{2}}{\partial r}\right|\right\}^{n-1 / 2}\right\} \frac{\partial u_{1}}{\partial r}\right. \\
& \left.\lambda_{2} \frac{\partial^{2} u_{1}}{\partial r \partial t}\right]+\mu_{0}\left(\left\{\frac{\mu_{\infty}}{\mu_{0}}+\left(1-\frac{\mu_{\infty}}{\mu_{0}}\right)\left\{1+\Gamma^{2}\left|\frac{\partial u_{1}^{2}}{\partial r}\right|\right\}^{n-1 / 2}\right\} \frac{\partial^{2} u_{1}}{\partial r^{2}}+\left(\mid 1-\frac{\mu_{\infty}}{\mu_{0}}\right) \frac{\partial}{\partial r}\left(\left\{1+\Gamma^{2}\left|\frac{\partial u_{1}}{\partial r}\right|\right\}^{n-1 / 2}\right)\right) \frac{\partial u_{1}}{\partial r} \\
& \left.+\lambda_{2} \frac{\partial^{3} u_{1}}{\partial r^{2} \partial t}\right),
\end{aligned}
$$

Similarly, substitution of Eqn. (13) into Eqn. (14) yields the determining equation for $u_{2}$ as follows:

$$
\rho_{2} \frac{\partial u_{2}}{\partial t}=A_{0}\left(1+\frac{A_{1}}{A_{0}} \cos \left(\omega_{p} t\right)\right)+\frac{\mu_{1}}{r} \frac{\partial u_{2}}{\partial r}+\mu_{2} \frac{\partial^{2} u_{2}}{\partial r^{2}},
$$

where subscripts denotes differentiation with respect to the indicated variable. Let us introduce the following variables

$$
\begin{aligned}
& \bar{r}=\frac{r}{a}, \bar{z}=\frac{z}{L} \bar{u}_{i}=\frac{u}{U_{0}}(i=1,2), \bar{t}=\frac{\omega_{p}}{2 \pi} t, U_{0}=\frac{A_{0} a^{2}}{\mu_{2}}, \bar{p}=\frac{a^{2} p}{\mu_{i} U_{0} L}(i=1,2), \\
& \bar{\lambda}_{1}=\frac{\lambda_{1} \omega_{p}}{2 \pi}, \bar{\lambda}=\frac{\lambda_{2} \omega_{p}}{2 \pi}, \bar{\Gamma}=\frac{\Gamma U_{0}}{a}, \rho^{*}=\frac{\rho_{1}}{\rho_{2}}, \alpha_{1}=\frac{\rho_{1} \omega_{p} a^{2}}{2 \pi \mu_{0}}, \alpha_{2}=\frac{\rho_{2} \omega_{p} a^{2}}{2 \pi \mu_{1}}=\left(\frac{\mu^{*}}{\rho^{*}}\right) \alpha_{1}, \\
& e=\frac{A_{1}}{A_{0}}, B_{1}=\frac{A_{0} a^{2}}{\mu_{0} U_{0}}, \widehat{B}_{1}=\frac{A_{0} a^{2}}{\mu_{1} U_{0}}=\mu^{*} B_{1}, \gamma=\frac{\mu_{\infty}}{\mu_{0}}, \beta=\frac{R_{1}}{a} .
\end{aligned}
$$

Making use of these variables, Eqns. (17)-(18) can be rendered into following dimensionless forms:

$$
\alpha_{1}\left(\frac{\partial u_{1}}{\partial t}+\lambda_{1} \frac{\partial^{2} u_{1}}{\partial t^{2}}\right)=B_{1}(1+e \cos (2 \pi t))-\left(2 \lambda_{1} e B_{1} \pi\right) \sin (2 \pi t)+\frac{1}{r}\left[\left\{\gamma+(1-\gamma)\left\{1+\Gamma^{2}\left|\frac{\partial u_{1}^{2}}{\partial r}\right|\right\}^{n-1 / 2}\right\} \frac{\partial u_{1}}{\partial r}+\lambda_{2} \frac{\partial^{2} u_{1}}{\partial r \partial t}\right]
$$




$$
\begin{gathered}
+\left(\left\{\gamma+(1-\gamma)\left\{1+\Gamma^{2}\left|\frac{\partial u_{1}^{2}}{\partial r}\right|\right\}^{n-1 / 2}\right\} \frac{\partial^{2} u_{1}}{\partial r^{2}}+\left((1-\gamma) \frac{\partial}{\partial r}\left(\left\{1+\Gamma^{2}\left|\frac{\partial u_{1}^{2}}{\partial r}\right|\right\}^{n-1 / 2}\right)\right) \frac{\partial u_{1}}{\partial r}+\lambda_{2} \frac{\partial^{3} u_{1}}{\partial r^{2} \partial t}\right) \\
\alpha_{2} \frac{\partial u_{2}}{\partial t}=\widehat{B}_{1}(1+e \cos (2 \pi t))+\frac{1}{r} \frac{\partial u_{2}}{\partial r}+\frac{\partial^{2} u_{2}}{\partial r^{2}}
\end{gathered}
$$

In the preceding equations the bar notation has been omitted for brevity. The above equations are subject to the following boundary and initial conditions:

$$
\left.u_{1 r}(r, t)\right|_{r=0}=0,\left.u_{1}(r, t)\right|_{\beta}=\left.u_{2}(r, t)\right|_{\beta},\left.u_{1 r}(r, t)\right|_{\beta}=\left.u_{2 r}(r, t)\right|_{\beta},\left.u_{2}(r, t)\right|_{r=R}=0, u_{1}(r, 0)=0,
$$

The first condition represents the symmetry of velocity $u_{1}$ with respect to the central plane. The next two conditions at $r=\beta$ indicate the continuity of velocity and stresses at the interface. The penultimate condition corresponds to the well-known no-slip initiation at the wall. The last condition represents the initiation of flow from the rest state.

The physical quantities of interest such as volumetric flow rate, wall shear stress (WSS) and resistance impendence in dimensionless form read:

$$
\begin{aligned}
& Q=\left(\int_{0}^{\beta} u_{1} r d r+\int_{\beta}^{1} u_{2} r d r\right), \\
& \tau_{w}=\left(\frac{\partial u_{2}}{\partial r}\right)_{r=R(z)} \cdot \\
& \Lambda=\frac{\left(\frac{\partial p}{\partial z}\right)}{Q},
\end{aligned}
$$

where

$$
\begin{aligned}
& R(z)=(1, \beta)-\frac{128}{5}(\delta, \tau)\left(\frac{11}{32}(z-\sigma)-\frac{47}{48}(z-\sigma)^{2}+(z-\sigma)^{3}-\frac{1}{3}(z-\sigma)^{4}\right), \quad \sigma \leq z \leq \sigma+\frac{3}{2}, \\
& \text { with } \quad \delta=\frac{\delta^{*}}{a}, \tau=\frac{\tau^{*}}{a}, \sigma=\frac{d}{l_{0}} .
\end{aligned}
$$

Employing a radial coordinate transformation [29]:

$$
x=\frac{r}{R(z)}
$$


Eqns. (20) and (21) can be transformed into the following equations:

$$
\begin{aligned}
& \alpha_{1}\left(\frac{\partial u_{1}}{\partial t}+\lambda_{1} \frac{\partial^{2} u_{1}}{\partial t^{2}}\right)=B_{1}(1+e \cos (2 \pi t))-\left(2 \lambda_{1} e B_{1} \pi\right) \sin (2 \pi t) \\
& +\frac{1}{x R^{2}}\left[\left\{\gamma+(1-\gamma)\left\{1+\left(\frac{\Gamma}{R}\right)^{2}\left|\frac{\partial u_{1}^{2}}{\partial x}\right|\right\}^{n-1 / 2}\right\} \frac{\partial u_{1}}{\partial x}+\lambda_{2} \frac{\partial^{2} u_{1}}{\partial x \partial t}\right]+\frac{1}{R^{2}}\left(\left\{\gamma+(1-\gamma)\left\{1+\left(\frac{\Gamma}{R}\right)^{2}\left|\frac{\partial u_{1}}{\partial x}\right|\right\}^{n-1 / 2}\right\} \frac{\partial^{2} u_{1}}{\partial x^{2}}\right. \\
& \left.+\left((1-\gamma) \frac{\partial}{\partial x}\left(\left\{1+\left(\frac{\Gamma}{R}\right)^{2}\left|\frac{\partial u_{1}}{\partial x}\right|\right\}^{n-1 / 2}\right)\right) \mid \frac{\partial u_{1}}{\partial x}+\lambda_{2} \frac{\partial^{3} u_{1}}{\partial x^{2} \partial t}\right) \\
& \alpha_{2}\left(\frac{\partial u_{2}}{\partial t}\right)=B_{1}(1+e \cos (2 \pi t))+\frac{1}{x R^{2}} \frac{\partial u_{2}}{\partial x}+\frac{1}{R^{2}} \frac{\partial^{2} u_{2}}{\partial x^{2}}
\end{aligned}
$$

The dimensionless initial and boundary conditions become:

$$
\left.u_{1}^{\prime}\right|_{x=0}=0,\left.\quad u_{2}\right|_{x=1}=0,\left.u_{1}\right|_{\beta}=\left.u_{2}\right|_{\beta},\left.u_{1}^{\prime}\right|_{\beta}=\left.u_{2}^{\prime}\right|_{\beta},\left.\quad u_{k}\right|_{t=0}=0, k=1,2
$$

Similarly the volume flow rate, shear stress at the wall and resistance (impedance), respectively, assume the following forms:

$$
\begin{aligned}
Q & =R^{2}\left(\int_{0}^{\beta} u_{1} x d x+\int_{\beta}^{1} u_{2} x d x\right), \\
\tau_{w} & =\frac{1}{R}\left(\frac{\partial u_{2}}{\partial x}\right)_{x=1} . \\
\Lambda & =\frac{\left(\frac{\partial p}{\partial z}\right)}{Q} .
\end{aligned}
$$

Substituting the dimensionless form of the pressure gradient into Eqn. (33), we may write:

$$
\Lambda=\frac{B_{1}(1+e \cos (2 \pi t))}{\left(\int_{0}^{\beta} u_{1} x d x+\int_{\beta}^{1} u_{2} x d x\right) R^{2}(z)}
$$




\section{EXPLICIT FINITE DIFFERENCE METHOD (FDM) SOLUTIONS}

The parabolic system of equations (28) and (29) is not amenable to analytical solution due to its nonlinear nature. Therefore, we shall employ an appropriate numerical technique for its solution. An explicit scheme which is forward in time and central in space is used [30-32]. Let us denote $u_{k i}^{j}$ as the value of $u_{k}(k=1,2)$ at spatial node $x_{i}$ and at time instant $t_{j}$. In this notation, the appropriate expressions of various partial derivatives are as follows:

$$
\begin{gathered}
\frac{\partial u_{k}}{\partial x} \cong \frac{u_{k i+1}^{j}-u_{k i-1}^{j}}{2 \Delta x}=u_{k x}, \\
\frac{\partial^{2} u_{k}}{\partial x^{2}} \cong \frac{u_{k i+1}^{j}-2 u_{k i}^{j}+u_{k i-1}^{j}}{(\Delta x)^{2}}=u_{k x^{2}} .
\end{gathered}
$$

In similar fashion, we approximate time derivative as:

$$
\begin{gathered}
\frac{\partial u_{k}}{\partial t} \cong \frac{u_{k i}^{j+1}-u_{k i}^{j}}{\Delta t}=u_{k t}, \\
\frac{\partial^{2} u_{k i}}{\partial t^{2}}=\frac{u_{k i}^{j+1}-2 u_{k i}^{j}+u_{k i}^{j-1}}{(\Delta t)^{2}}=u_{k t^{2}}, \\
\frac{\partial}{\partial t}\left(\frac{\partial u_{k}}{\partial x}\right) \cong \frac{u_{k i+1}^{j+1}-u_{k i+1}^{j}-u_{k i-1}^{j+1}+u_{k i-1}^{j}}{2 \Delta x \Delta t}=u_{k t x}, \\
\frac{\partial}{\partial t}\left(\frac{\partial^{2} u_{k}}{\partial x^{2}}\right) \cong \frac{u_{k i+1}^{j+1}-2 u_{k i}^{j+1}+u_{k i-1}^{j+1}-u_{k i+1}^{j}+2 u_{k i}^{j}-u_{k i-1}^{j}}{(\Delta x)^{2} \Delta t}=u_{k t x^{2}} .
\end{gathered}
$$

Using the above formulas for time and spatial derivatives, Eqns. (28) and (29) may be transformed in the following finite difference form:

$$
\begin{aligned}
u_{1 i}^{j+1}= & \frac{1}{\left(1+\frac{\lambda_{1}}{\Delta t}\right)}\left[u_{1 i}^{k}\left(1+\frac{2 \lambda_{1}}{\Delta t}\right)-\frac{\lambda_{1}}{\Delta t} u_{1 i}^{j-1}+\left(\frac{\Delta t}{\alpha_{1}}\right)\left[B_{1}\left(1+e \cos \left(2 \pi t^{j}\right)-\left(2 \lambda_{1} e \pi\right) \sin \left(2 \pi t^{j}\right)\right)+\right.\right. \\
+ & \frac{1}{x R^{2}}\left[\left\{\gamma+(1-\gamma)\left\{1+\left(\frac{\Gamma}{R}\right)^{2}\left|u_{1 x}^{2}\right|\right\}^{n-1 / 2}\right\} u_{1 x}+\lambda_{2} u_{1 x t}\right]+\frac{1}{R^{2}}\left(\left\{\gamma+(1-\gamma)\left\{1+\left(\frac{\Gamma}{R}\right)^{2}\left|u_{1 x}^{2}\right|\right\}^{n-1 / 2}\right\} u_{1 x^{2}}\right. \\
& +\left((1-\gamma) \frac{\partial}{\partial x}\left(\left\{1+\left(\frac{\Gamma}{R}\right)^{2}\left|u_{1 x}^{2}\right|\right\}^{n-1 / 2}\right) \mid u_{1 x}+\lambda_{2} u_{1 t x^{2}}\right),(37)
\end{aligned}
$$




$$
u_{2 i}^{j+1}=u_{2 i}^{j}+\left(\frac{\Delta t}{\alpha_{2}}\right)\left[\hat{B}_{1}\left(1+e \cos \left(2 \pi t^{j}\right)\right)+\frac{1}{x R^{2}}\left(u_{2 x}\right)+\frac{1}{R^{2}} u_{2 x^{2}}\right] .
$$

The finite difference representation of the associated prescribed boundary conditions is given by:

$$
\begin{array}{ll}
u_{i}^{1}=0, & \text { at } t=t_{1}=0 \\
u_{1 N_{c}+1}^{j}=u_{2 N_{c}+1}^{j}, & \text { at } x=x_{N_{c}+1}=\beta \\
u_{N+1}^{k}=0, & \text { at } x=1 \\
u_{1}^{k}=u_{2}^{k} & \text { at } x=0 .
\end{array}
$$

The uniformly distributed discrete points in the radial direction are defined as $x_{i}=(i-1) \Delta x$, $\left(i=1,2, \ldots, N_{c}+1\right)$ such that $x_{\left(N_{c}+1\right)}=\beta$ and $x_{i}=\left(i-\left(N_{c}+1\right)\right) \Delta x, i=\left(N_{c}+1, N_{c}+2, \ldots, N+1\right)$ such that $x_{N+1}=1$, where $\Delta x$ is the increment in the radial direction. Similarly we define $t_{j}=(j-1) \Delta t,(j=1,2, \ldots)$ as discrete time points with $\Delta t$ indicating a small time increment. At a particular cross-section the simulations are carried out for a specific value of temporal and spatial step sizes $\Delta t=\Delta t_{1}$ and $\Delta x=\Delta x_{1}$. Indeed, it is anticipated that for this specific choice the numerical values of velocity might not be convergent. This claim can be verified by choosing lower values of $\Delta t=\Delta t_{2}\left(<\Delta t_{1}\right)$ and $\Delta x=\Delta x_{2}\left(<\Delta x_{1}\right)$ and then comparing the numerical value of velocity with the previously obtained values. Now, if the absolute difference between the corresponding numerical values is less than the prescribed tolerance, say $10^{-7}$, then it can be inferred that the results are accurate up to 7 decimal places for $\Delta t=\Delta t_{1}$ and $\Delta x=\Delta x_{1}$. For the present problem an accuracy of $10^{-7}$ is achieved by taking $\Delta t=$ 0.00001 and $\Delta x=0.025$.

\section{VALIDATION WITH VARIATIONAL FINITE ELEMENT CODE}

The results obtained via the FDM computations are also validated by the finite element method. The nonlinear boundary value problem described by the coupled partial differential eqns. (28)-(29) under conditions (30) has also been solved with a variational finite element method (FEM). FEM has a different approach to FDM. Numerical integration is employed rather than numerical differentiation. Owing to this and the adaptability of FEM to complex material and geometrically nonlinear problems, FEM remains the most popular numerical method in engineering sciences. In nonlinear biomechanics, the FEM approach has successively resolved numerous problems in diverse areas including peristaltic pumping [33], biomagnetic therapy [34], bio-membrane structural stability [35], functional graded implant mechanics [36], pulsatile non-Newtonian flows [37] and respiratory fluid dynamics [38]. Pressure gradient is re- 
defined and time conditions (temporal) also re-defined. Following some numerical tests, meshindependence is confirmed for the present scenario with 480 line finite elements. The whole domain is therefore divided into a set of 480 line elements of equal width, each element being two-noded. Line elements are adequate since only one spatial variable i.e. normalized radial coordinate, $(x)$ is involved. A variational form is derived for each of the transport eqns. (28)-(29) with the lead master variables $u_{1}, u_{2}$. The numerical integration is performed over the artificial finite element domain in terms of the normalized radial coordinate $\left(x_{e} ; x_{e}+1\right)$ using arbitrary test functions $\left(W_{l}, W_{2}\right)$ which can be viewed as the variation in the master variables, following Reddy [39] and Bathe [40]. The nonlinear terms in eqns. (28)(29) are easily accommodated. The finite element form of the variational equations is achieved by appropriate substitutions based on the following approximations:

$u_{1}=\sum_{j=1}^{2} u_{2 j} \psi_{j} ; u_{2}=\sum_{j=1}^{2} u_{1 j} \psi_{j}$

With the following definitions for weighting functions [39, 40]:

$$
W_{1}=W_{2}=\psi_{i}, \quad i=1,2
$$

The shape (interpolation) functions for a typical line element $\left(x_{e}, x_{e+1}\right)$ in eqn. (56) are prescribed as follows:

$\psi_{1}^{(e)}=\frac{x_{e+1}-x}{x_{e+1}-x_{e}} ; \psi_{2}^{(e)}=\frac{x-x_{e}}{x_{e+1}-x_{e}}, x_{e} \leq x \leq x_{e+1}$

The matrix-vector form of the finite element model is then generated. For brevity details are excluded here and the reader is referred to [33]-[35] wherein comprehensive guidance is provided. This system of non-linear algebraic equations produced after assembly of the element equations is linearized by incorporating functions $u_{1}, u_{2}$, which are assumed to be known. Boundary conditions (30) are also easily constructed. Following imposition of the initial (time) and boundary conditions, the matrix system is condensed and solved iteratively with a modified Householder elimination method, maintaining an accuracy of 0.0005 . For extra rapid convergence the Bathe subspace iteration procedure may also be employed [40]. Table 1 shows that very good agreement is attained between the FEM and FDM results. Confidence is therefore very high in the present FDM computations. Table 1 further provides a useful benchmark for other researchers to refer to in refinements to the present investigation. 


\begin{tabular}{|c|c|c|c|c|}
\hline \multirow{2}{*}{$x$} & \multicolumn{5}{|c|}{ Numerical values of axial velocity } \\
& $n=0.7$ & $n=0.7$ & $n=1$ & $n=1$ \\
& FDM & FEM & FDM & FEM \\
\hline 0 & 1.0648 & 1.0647 & 0.9079 & 0.9077 \\
\hline 0.1000 & 1.0566 & 1.0564 & 0.8995 & 0.8994 \\
\hline 0.2000 & 1.0276 & 1.0275 & 0.8702 & 0.8701 \\
\hline 0.3000 & 0.9778 & 0.9776 & 0.8214 & 0.8212 \\
\hline 0.4000 & 0.9060 & 0.9059 & 0.7535 & 0.7536 \\
\hline 0.5000 & 0.8110 & 0.8109 & 0.6674 & 0.6672 \\
\hline 0.6000 & 0.6920 & 0.6918 & 0.5639 & 0.5638 \\
\hline 0.7000 & 0.5483 & 0.5485 & 0.4442 & 0.4441 \\
\hline 0.8000 & 0.3799 & 0.3801 & 0.3096 & 0.3095 \\
\hline 0.9000 & 0.1938 & 0.1939 & 0.1609 & 0.1610 \\
\hline 1.0000 & 0 & 0 & 0 & 0 \\
\hline
\end{tabular}

Table 1: Numerical values of axial velocity at a cross-section $z=0.79$ corresponding to the critical height of the stenosis for $\lambda_{1}=0.3, \lambda_{2}=0.1, \Gamma=1, \delta=0.1, t=1.15$.

\section{RESULTS AND INTERPRETATION}

In this section, we are interested in establishing the response of the axial velocity, flow rate, wall shear stress and resistance (impedance) to flow for different values of non-dimensional parameters namely the relaxation and retardation times $\left(\lambda_{1}\right.$ and $\left.\lambda_{2}\right)$, geometrical parameter $(\beta)$, time constant $(I)$ and power law parameter $(n)$. In all numerical computations, the following set of default parameters are used, following Yilmaz and Gundogdu [1]:

$l_{0}=1, \beta=0.8, L=2.5, \alpha_{1}=\alpha_{2}=0.5, \mu_{0}=0.56, \mu_{\infty}=0.0345, \phi=0.0, \sigma=0.5$. This data accurately corresponds to realistic biorheological hemodynamics.

Figs. 2-4 demonstrate the dimensionless velocity profiles of blood at the stenotic throat of the arterial segment under study. The effects of power law index $(n)$ and time constant $(I)$ on axial velocity are shown in Figs. 2a and $\mathbf{2 b}$, respectively. Fig. 2a shows that the magnitude of axial velocity reduces significantly by increasing $n$. The boost in blood viscosity with greater power-law exponent serves to decrease the shear rate and this decelerates the flow, manifesting in a reduction in velocity. On the contrary, the axial velocity of blood increases with increasing $(\Gamma$ ) (Fig. 2b) since the delay in material 
response is reduced with greater time constant. In both plots the acceleration in axial velocity with greater radial coordinate is however apparent.
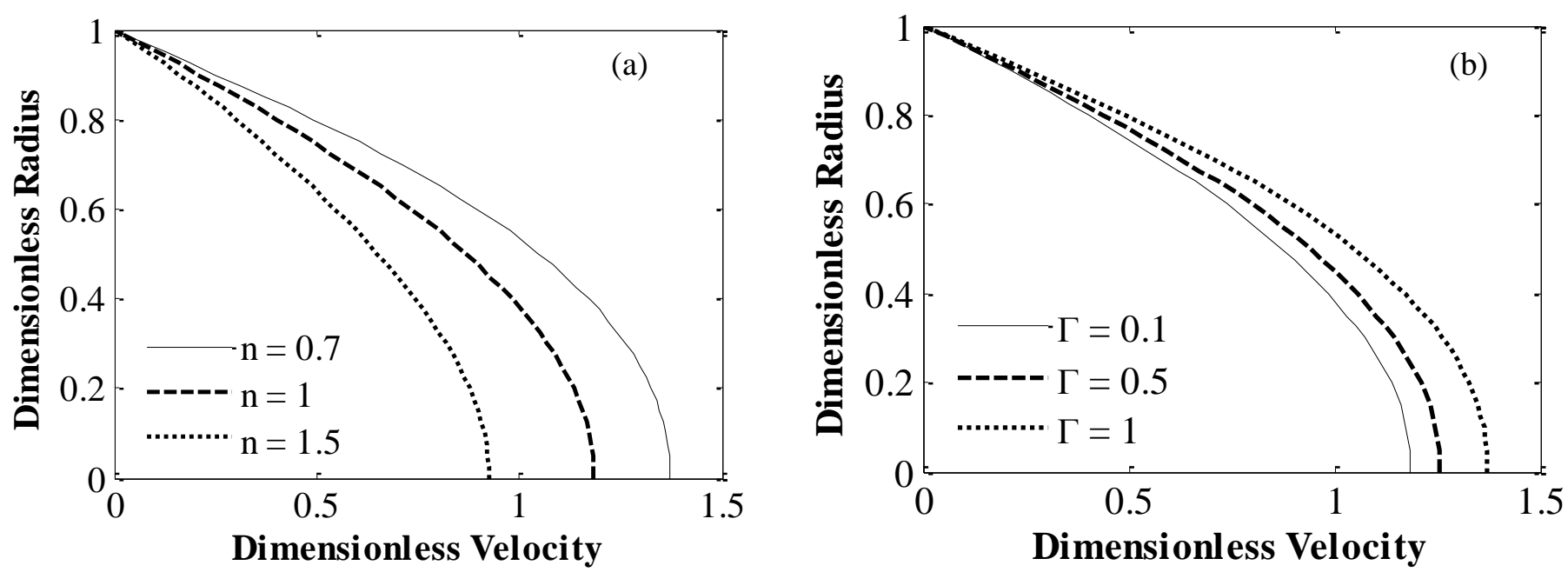

Fig. 2. Variation of velocity with radial distance for

$$
\left(\alpha=0.5, \beta=0.8, \mathrm{~B}_{1}=4, \lambda_{1}=0.3, \lambda_{2}=0.1, \mathrm{t}=\tau_{s}, \Gamma=1, n=0.7, \mathrm{e}=0.3\right) .
$$

Fig. 3 illustrates the effect of relaxation and retardation time constants on the axial velocity of blood. Panel (a) indicates that the magnitude of dimensionless velocity profiles at the stenotic throat of the arterial segment is a decreasing function of $\lambda_{2}$. Panel (b) reveals that the axial velocity of blood follows the converse trend with increasing $\lambda_{1}$. The influence of both Oldroyd-B parameters (viscoelastic) is clearly non-trivial. This emphasizes the need to include rheological characteristics in mathematical models of blood flow, and confirms the inadequacy of the Newtonian model.
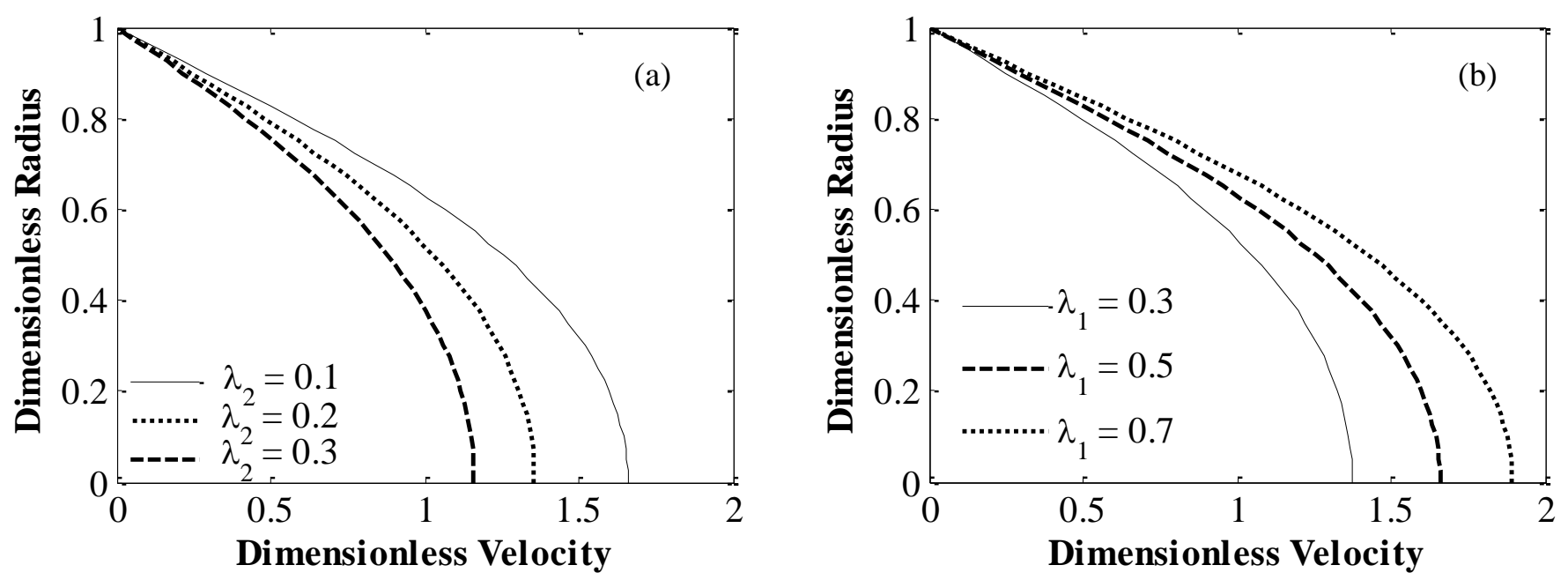

Fig. 3. Variation of velocity with radial distance for $\left(\alpha=0.5, \beta=0.8, \mathrm{~B}_{1}=4, \lambda_{1}=0.5, \lambda_{2}=0.1, \mathrm{t}=\tau_{s}, \Gamma=1, n=0.7, \mathrm{e}=0.3\right)$. 


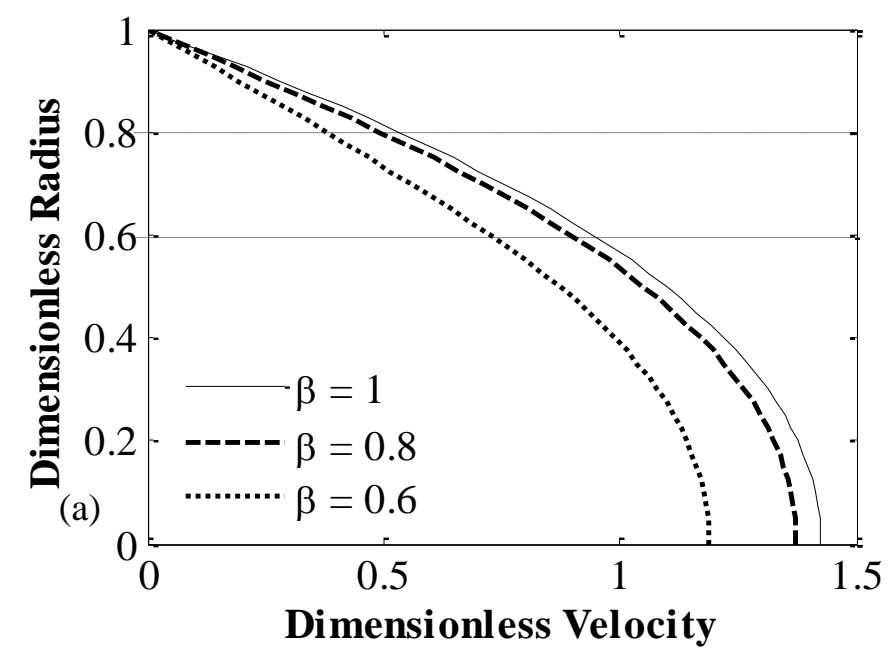

Fig. 4. Variation of velocity with radial distance for $\left(\alpha=0.5, \mathrm{~B}_{1}=4, \lambda_{1}=0.5, \lambda_{2}=0.2, \mathrm{t}=\tau_{s}, \Gamma=1, n=0.7, \mathrm{e}=0.3\right)$.

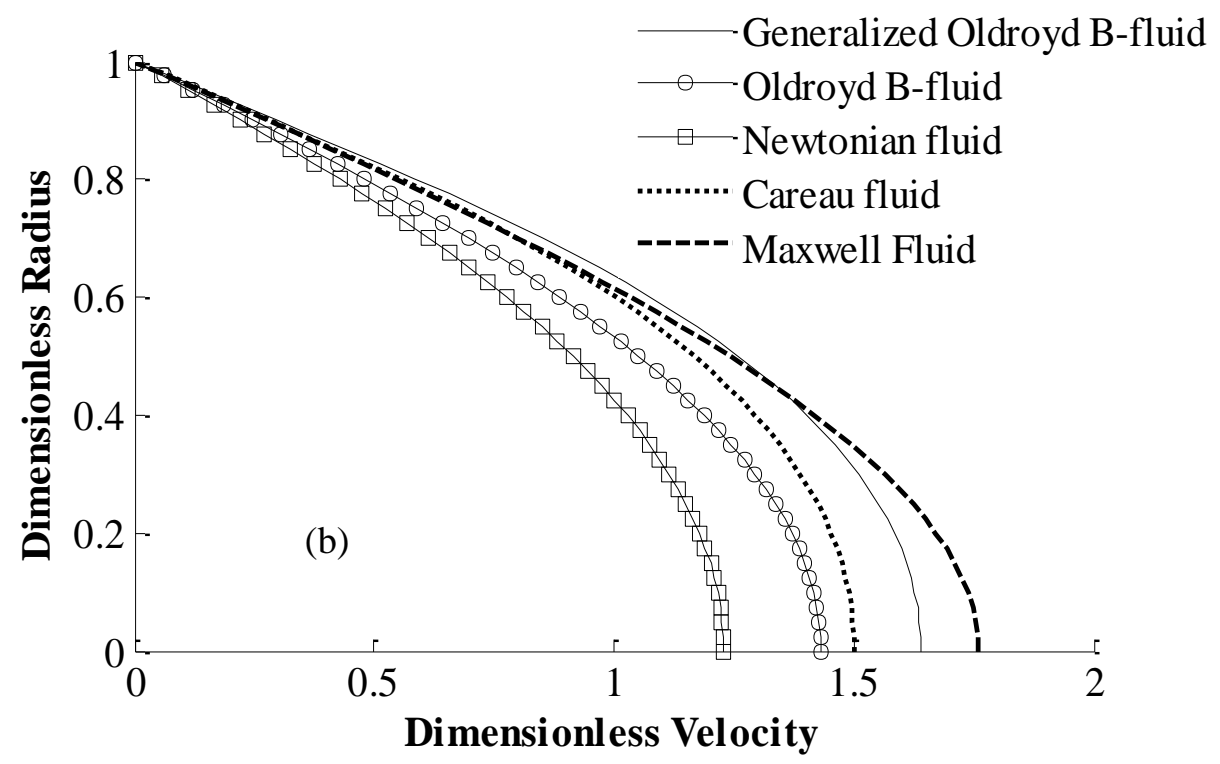

Fig. 5. Variation of velocity with radial distance for Maxwell fluid $\left(\lambda_{1}=0.5\right)$, Oldroyd B-fluid $\left(\lambda_{1}=0.5, \lambda_{2}=0.2\right)$, Carreau fluid $(\Gamma=1, n=0.7)$, Newtonian fluid and generalized Oldroyd-B fluid

$$
\left(\lambda_{1}=0.5, \lambda_{2}=0.2, \Gamma=1, n=0.7\right) \text {. }
$$

The geometric parameter $\beta$ can be used to investigate the effect of peripheral layer thickness on axial velocity of the flowing blood. To this end, the velocity distribution for different values of the interface position $\beta$ is shown in Fig. 4. It is found that the velocity decreases substantially by increasing peripheral layer thickness. The profiles of axial velocity of blood based on different fluid models are shown in Fig. 5. It is evident that the Maxwell fluid model predicts the highest values of axial velocity whilst the Newtonian fluid model predicts the least values amongst all models. Nevertheless the non- 
Newtonian models all predict axial velocity magnitudes greater than the Newtonian model. The latter is therefore insufficient and under-predicts the flow response considerably.

Fig. 6 depicts the variation of flow rate for different values of $\lambda_{1}$ and $\lambda_{2}$ when $B=4, e=0.5$ and $\beta=$ 0.8. Panel 6(a) indicates an elevation in flow rate as $t$ increases from 0 to 2.1. However, after $t=2.1$, steady state conditions are observed to prevail. i.e., flow rate becomes periodic rather than erratic. In such situations it is found that the amplitude of flow rate increases by increasing $\lambda_{1}$. Moreover, a phase shift occurs with the increase of $\lambda_{1}$. Fig. 6(b) reveals that the amplitude of flow rate decreases with $\lambda_{2}$. The effect of inducing a phase shift by increasing $\lambda_{2}$ can also be observed from Fig. 6(b).

The evolution of flow rate with time for various values of power law index $(n)$ and time constant of Carreau model $(\Pi)$ is shown through Fig. 7. It is evident from Fig. 7(a) that the amplitude of oscillations in the flow rate diminishes by increasing the power law index. On the contrary, oscillation amplitude is found to increase with greater values of $\Gamma$ (Fig. 7(b)).

The profiles of flow rate corresponding to different fluid models are compared in Fig. 8. Here similar to velocity plots, it is observed that amongst all models, the Maxwell model predicts higher values of flows rate. The values of flow rate predicted by the Newtonian model are the lowest computed. It is however important to highlight that some of the values of flow rate predicted by the Maxwell model after achieving steady state in systolic phase are zero or negative and thus might be unrealistic.
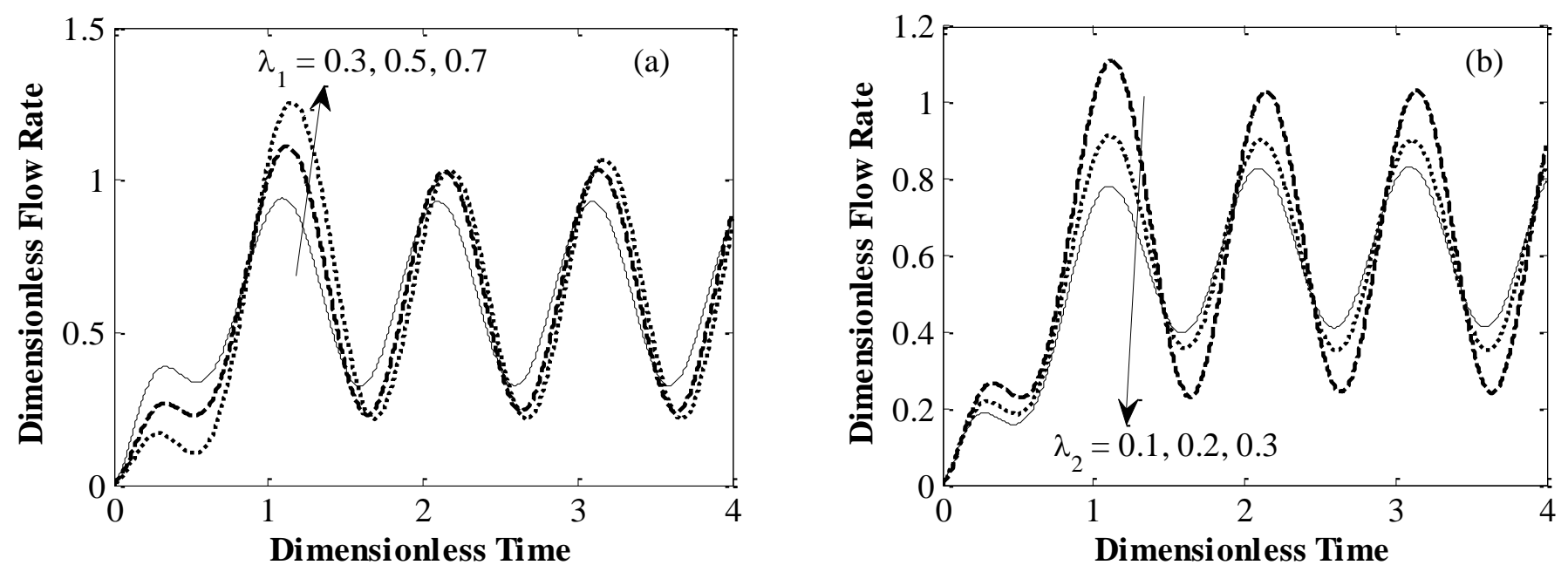

Fig. 6. Time series of flow rate for

$$
\left(\alpha=0.5, \beta=0.8, \mathrm{~B}_{1}=4, \lambda_{1}=0.5, \lambda_{2}=0.1, \mathrm{t}=\tau_{s}, \Gamma=1, n=0.7, \mathrm{e}=0.3\right) .
$$



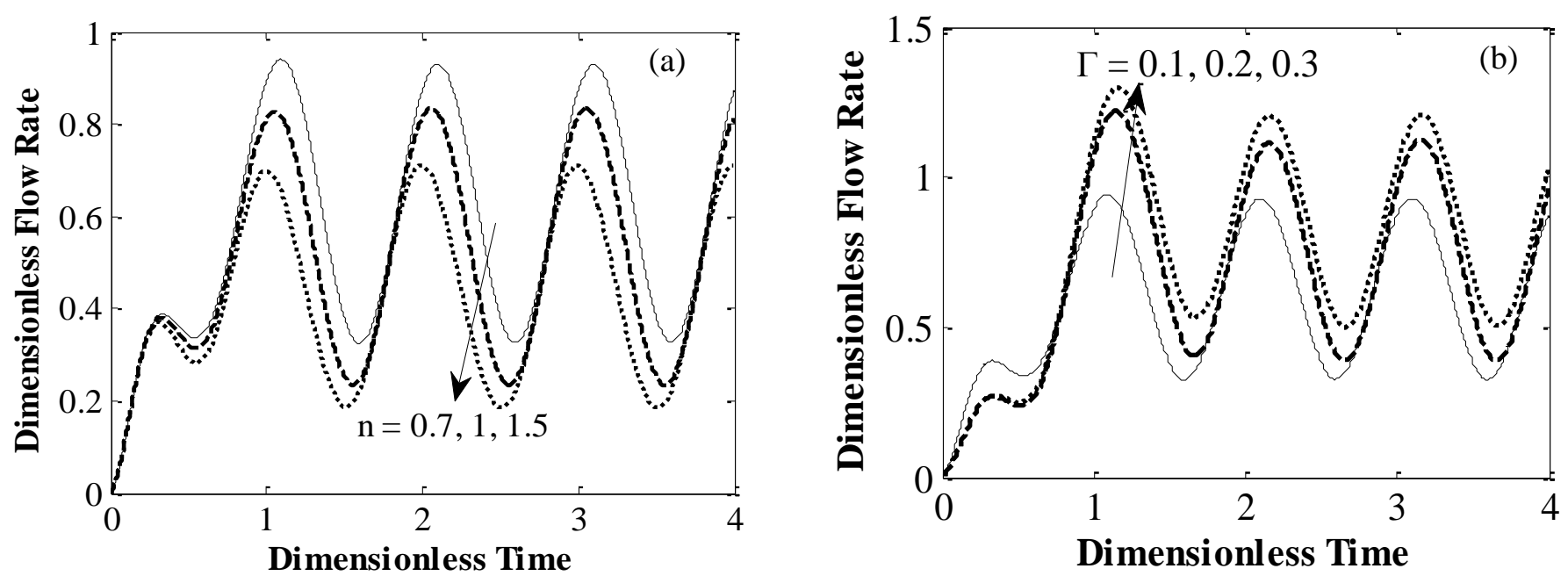

Fig. 7. Time series of flow rate for $\left(\alpha=0.5, \beta=0.8, \mathrm{~B}_{1}=4, \lambda_{1}=0.5, \lambda_{2}=0.1, \mathrm{t}=\tau_{s}, \Gamma=1, n=0.7, \mathrm{e}=0.3\right)$.

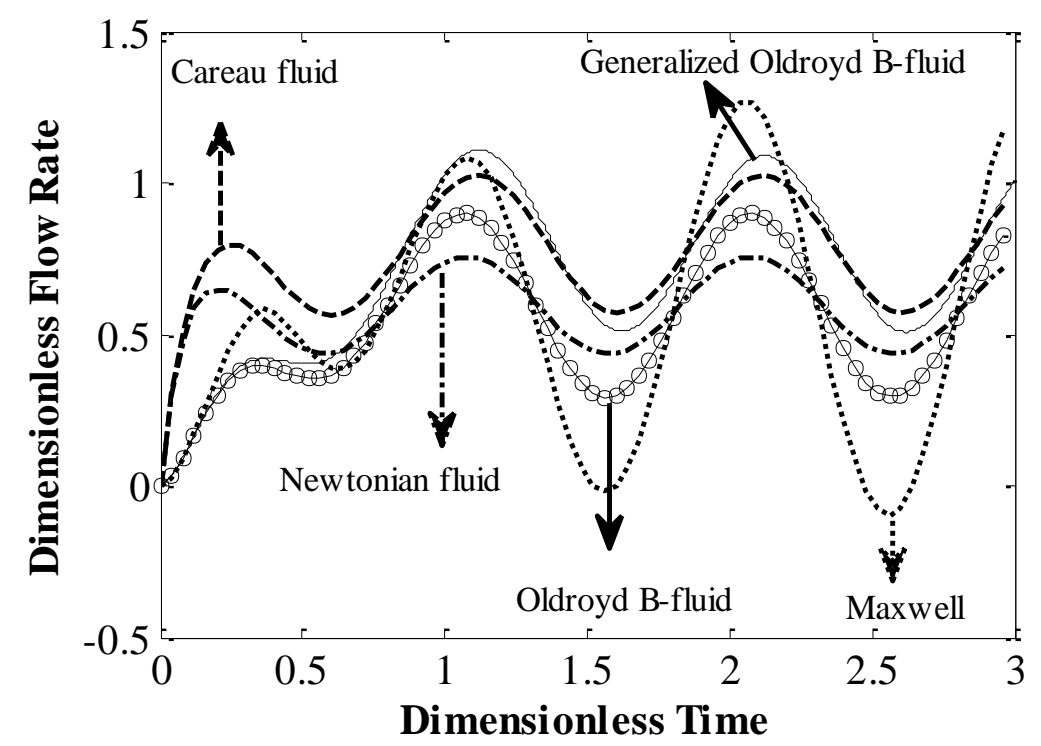

Fig. 8. Time-series of flow rate for $\operatorname{Maxwell}$ fluid $\left(\lambda_{1}=0.5\right)$, Oldroyd B-fluid $\left(\lambda_{1}=0.5, \lambda_{2}=0.2\right)$, Carreau fluid $(\Gamma=1, n=0.7)$, Newtonian fluid and generalized Oldroyd-B fluid $\left(\lambda_{1}=0.5, \lambda_{2}=0.2, \Gamma=1, n=0.7\right)$. 

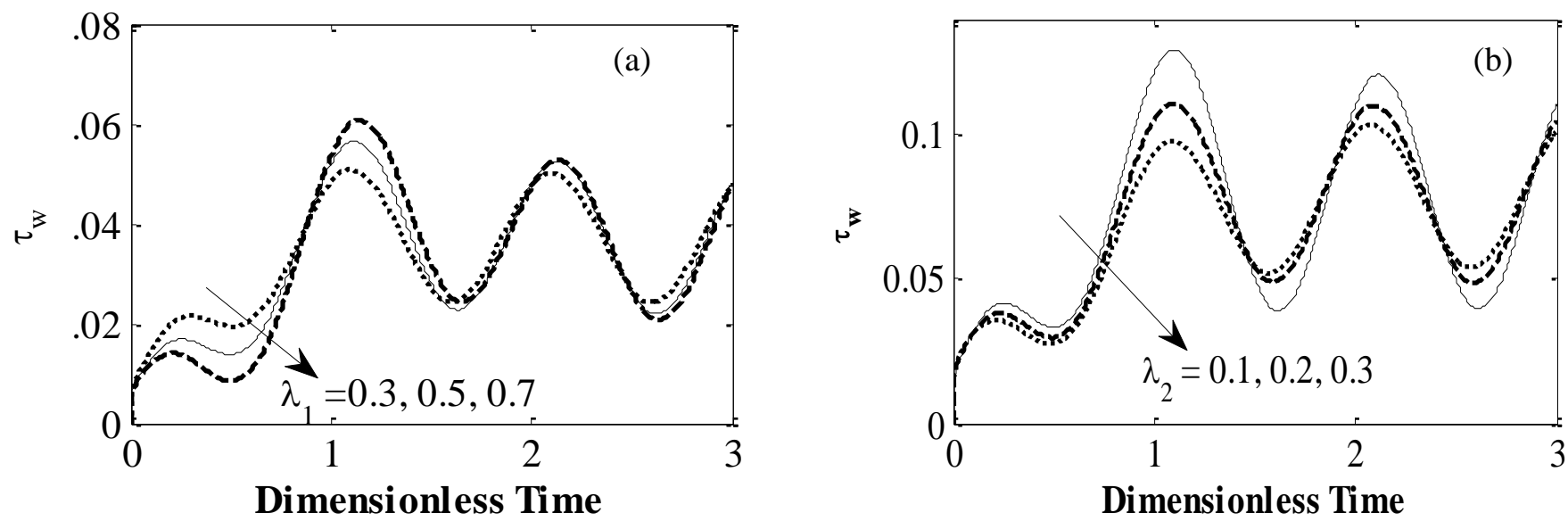

Fig. 9. Time series of wall shear stress (WSS) for

$\left(\alpha=0.5, \beta=0.8, \mathrm{~B}_{1}=4, \lambda_{1}=0.5, \lambda_{2}=0.1, \mathrm{t}=\tau_{s}, \Gamma=1, n=0.7, \mathrm{e}=0.3\right)$.

Fig. 9 shows the variation of wall shear stress in a cycle of oscillation for different values of $\lambda_{1}$ and $\lambda_{2}$ with $\beta=0.8$. This figure indicates that the magnitude of wall shear stress increases by increasing $\lambda_{1}$ (panel (a)) while it is suppressed by increasing $\lambda_{2}$. Moreover it is observed through Fig. 10 that the magnitude of the wall shear increases with an increase in the magnitude of the time constant $(I)$. The longitudinal impedance $(\Lambda)$ of the artery is calculated using Eq. (34) and its variation during a flow cycle for different values of $\lambda_{1}$ and $\Gamma$ with $e=0.3$ and $\beta=0.8$ is illustrated in Fig. 11. It is observed that these profiles follow the opposite trend as compared to the flow rate profiles. In fact it is found that the magnitude of resistance to flow increases with an increase in $\lambda_{1}$ whereas it exhibits the converse behavior with increasing $\Gamma$. The impedance curves for different fluid models are compared in Fig. 12. From this figure it can be deduced that the impedance is minimized for a generalized Oldroyd-B fluid whereas it is maximized for a conventional Oldroyd-B fluid. The impedance curve corresponding to the Maxwell model contains infinite jumps and therefore it is not shown here. These infinite jumps are the consequence of zero flow rate predicted by Maxwell model as shown in Fig. 8. Evidently the Maxwell model has serious shortcomings limiting its applicability in hemodynamics. 


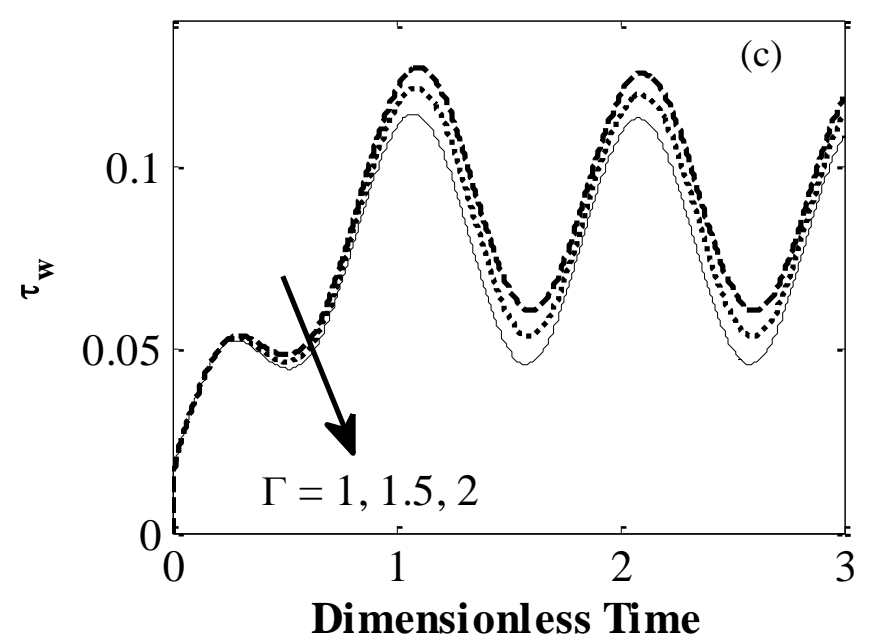

Fig. 10. Time-series of wall shear stress (WSS) for $\left(\alpha=0.5, \beta=0.8, \mathrm{~B}_{1}=4, \lambda_{1}=0.5, \lambda_{2}=0.1, \mathrm{t}=\tau_{s}, n=0.7, \mathrm{e}=0.3\right)$.
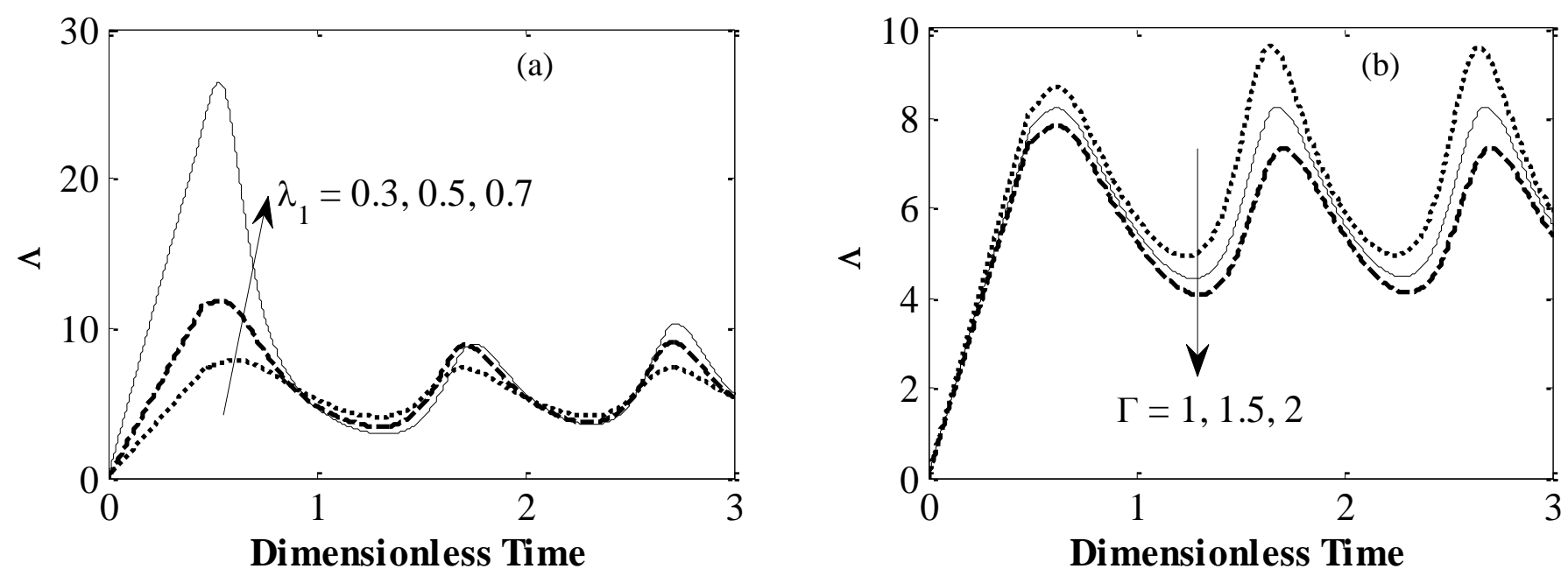

Fig. 11. Impedance plotted against time for $\left(\alpha=0.5, \beta=0.8, \mathrm{~B}_{1}=4, \lambda_{1}=0.5, \lambda_{2}=0.1, \mathrm{t}=\tau_{s}, \Gamma=1, n=0.7\right.$, $\left.\mathrm{e}=0.3\right)$. 


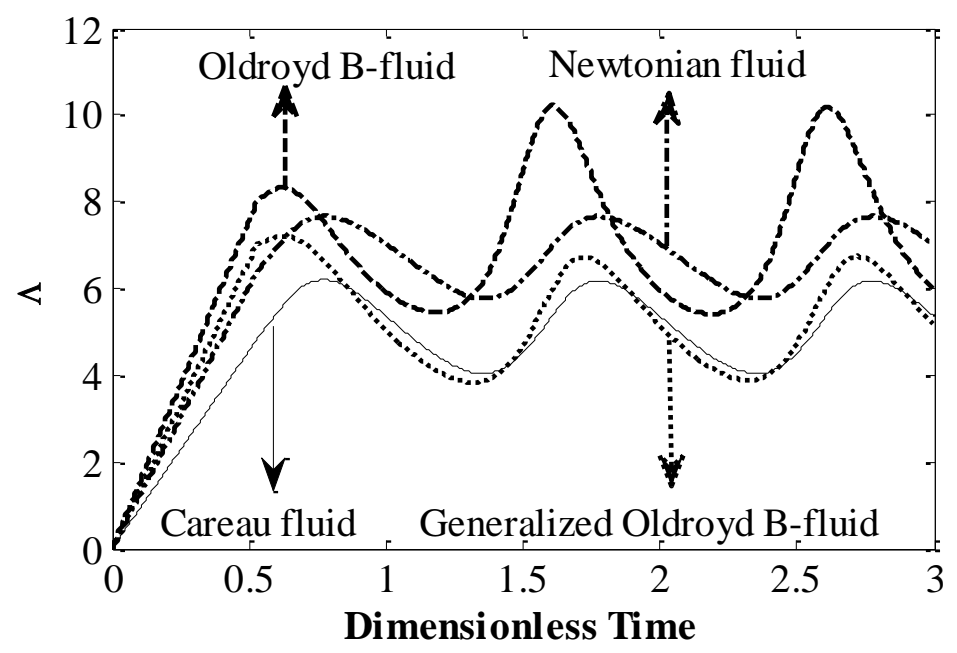

Fig. 12. Time-series of impedance for Oldroyd B-fluid $\left(\lambda_{1}=0.5, \lambda_{2}=0.2\right)$, Carreau fluid $(\Gamma=1, n=0.7)$, Newtonian fluid and generalized Oldroyd-B fluid $\left(\lambda_{1}=0.5, \lambda_{2}=0.2, \Gamma=1, n=0.7\right)$.

The instantaneous blood flow patterns for different values of the emerging parameters are compared in Fig. 13. Panel (a) shows the flow pattern for specific values of $\Gamma, \lambda_{1}$ and $\lambda_{2}$. This panel confirms the appearance of a circulating bolus of fluid in the stenotic region. It is evident from panel (b) that both size and circulation of bolus decrease by increasing $\lambda_{1}$ from 0.5 to 0.7 .
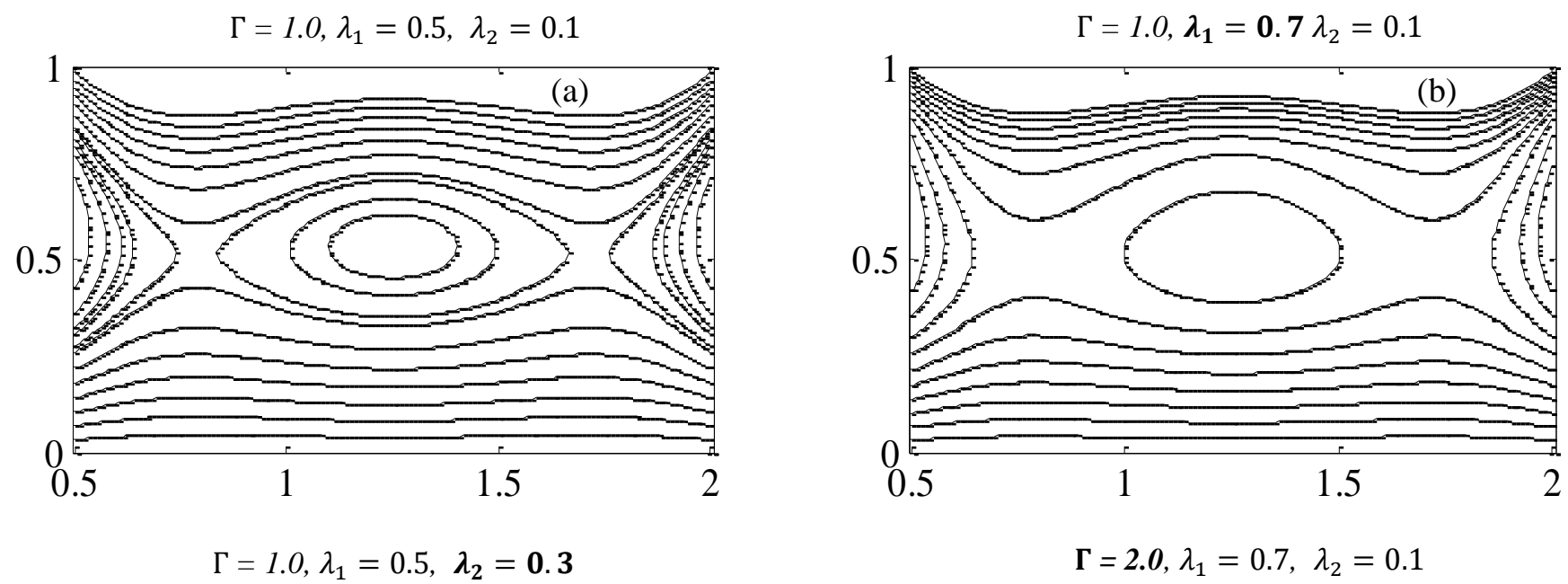

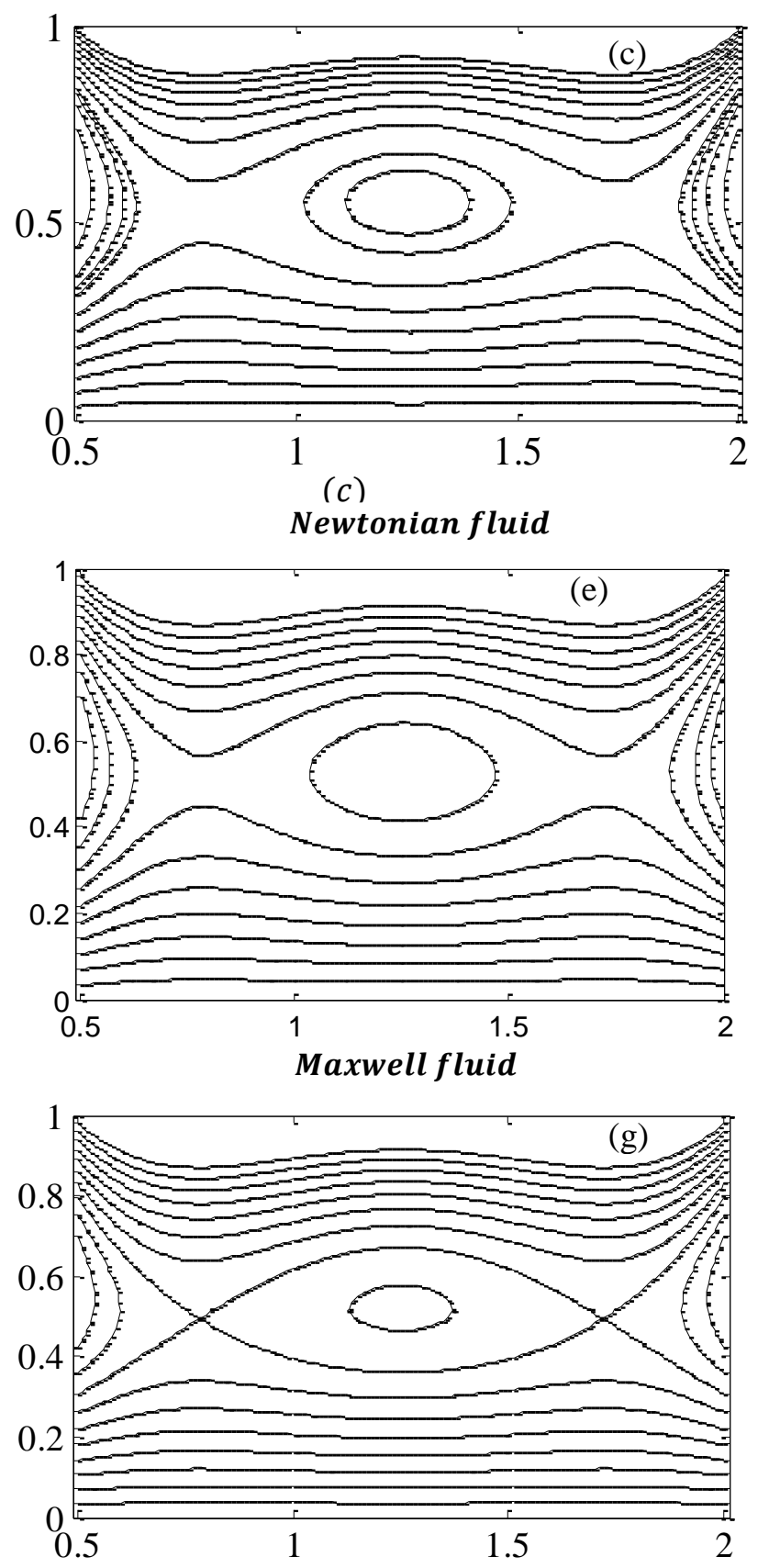
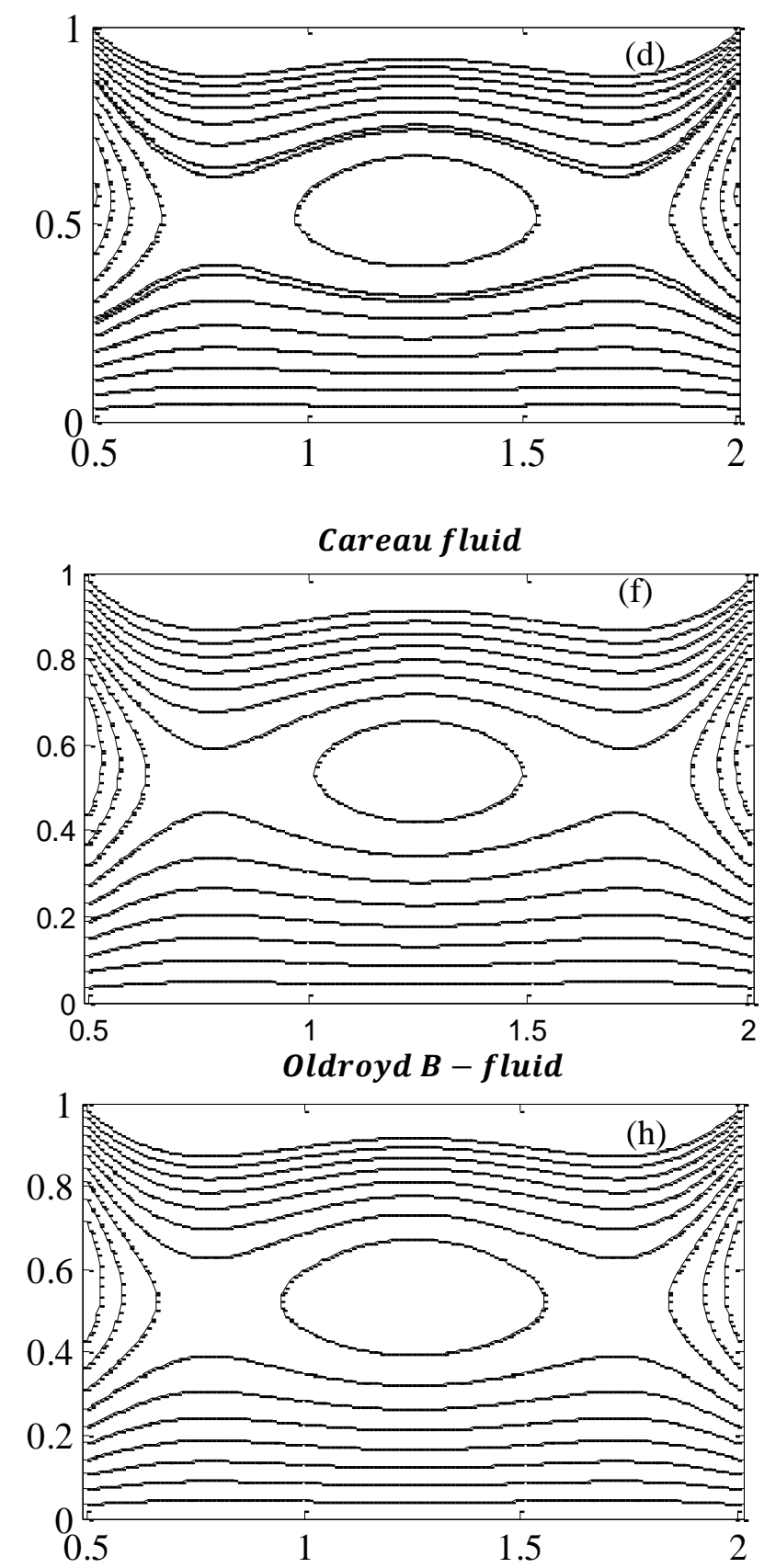

Fig. 13. Flow patterns with the following set of values $\left(\alpha=0.5, \beta=0.8, \mathrm{~B}_{1}=4, \mathrm{t}=0.4, \delta=0.1, n=0.7, \mathrm{e}=0.3\right)$.

A comparison of panel (a) and panel (c) discloses that both size and circulation of bolus are reduced by increasing $\lambda_{2}$ from 0.1 to 0.3 . The effects of $\Gamma$ on a circulating bolus of fluid can be observed by comparing panel (a) and panel (d). It is observed that the strength of circulating region decreases by increasing $\Gamma$. The relative flow patterns of blood predicted by the different rheological models can be made by comparing panel (a) with panels (e)-(h). Panel (a) shows the instantaneous streamlines of blood 
flow based on the generalized Oldroyd-B model. It is noted through the comparison of panel (a) with panel (e) that the strength of the recirculating zone (size and circulation of trapped bolus of fluid) appearing in the stenotic region decreases from the generalized Oldroyd-B fluid case to the Newtonian fluid case. Similarly, the other fluid models also predict a decrease in the size and circulation of trapped bolus of fluid in comparison with the generalized Oldroyd-B model.

\section{CONCLUDING REMARKS}

A two-layered mathematical model has been developed for pulsatile flow of blood through a $w$-shape stenosed artery using a generalized Oldroyd-B fluid model in the core region and a Newtonian model in the peripheral region. The analysis is based on the solution of a non-linear partial differential equation corresponding to each region. Assuming the continuity of velocity at interface and no-slip conditions, the governing equations are solved in each region by employing a finite difference technique. Verification of the accuracy of FDM solutions is confirmed with a finite element method (FEM). It is found that the resistance to flow (impedance), axial velocity, flow rate and wall shear stress are greatly affected by the rheological parameters featured in the generalized Oldroyd-B model. The flow characteristics of blood computed by applying different non-Newtonian models can also be compared through the simulation procedure documented herein. The present computations have identified that the Maxwell model predicts the highest magnitude of axial velocity amongst all other models, including the Newtonian model. The magnitude of axial velocity is least for the Newtonian model. In the steady state situation, the magnitude of flow rate is highest for the generalized Oldroyd-B model and least for the conventional Oldroyd-B model. It is also observed that the flow rate computed using the Maxwell model becomes negative at certain time instants belonging to the systolic phase. Such negative values of flow rate predicted by the Maxwell model are unrealistic as they are indicative of back flow. The impedance (resistance to flow) predicted by the generalized Oldroyd-B model is the lowest whereas it is the highest for the conventional Oldroyd-B model. The recirculating zone appears in the stenotic region for all non-Newtonian models including the generalized Oldroyd-B model. However, its strength is greatest in the case of the generalized Oldroyd-B model and weakest for the Maxwell model. The analysis presented here also highlights the effect of peripheral layer thickness on axial velocity of the blood. Additionally the computations show that axial velocity significantly decreases with an increase in the peripheral layer thickness. The present study has ignored curvature of the blood vessel and also elastic wall properties. These are also key aspects of hemodynamics and will be addressed in future investigations. 


\section{ACKNOWLEDGEMENTS}

The authors are grateful to the reviewer for his/her judicious comments, which have improved the present investigation. The first author is grateful to the Higher Education Commission (HEC) for financial assistance.

\section{REFERENCES}

[1]. F. Yilmaz, and M. Y. Gundogdu, A critical review on blood flow in large arteries; relevance to blood rheology, viscosity models, and physiologic conditions, Korea-Australia Rheo. J., 20, pp. 197-211, (2008).

[2]. P. K. Mandal, An unsteady analysis of non-Newtonian blood through tapered arteries with a stenosis, Int. J. Nonlinear. Mech. 40, 151-164 (2005).

[3] A. Zaman, N. Ali, M. Sajid, and T. Hayat, Effects of unsteadiness and non-Newtonian rheology on blood flow through a tapered time-variant stenotic artery, AIP Advances 5, 037129 (2015).

[4]. Y. C. Fung, Biomechanics: Mechanical Properties of Living Tissues, MacGraw-Hill, New York (1993).

[5]. B. M. Johnston, P. R. Johnston, S. Corney and D. Kilpatrick, Non-Newtonian blood flow in human right coronary arteries: steady state simulations, J. Biomech. 37, 709-720 (2004).

[6]. Y. I. Cho and K. R. Kensey, Effects of the non-Newtonian viscosity of blood on hemodynamics of diseased arterial flows: part 1, steady flows, Biorheology, 28, 241-262 (1991).

[7]. Y. Sugiura, A method for analyzing non Newtonian blood viscosity data in low shear rates, Biorheology 25, 107-112 (1988).

[8]. C. R. Huang, W. D. Pan, H. Q. Chen and A. L. Copley, Thixotropic properties of whole blood, Biorheology, 24, 795-801 (1987).

[9]. M. A. Rao, Rheology of Fluids and Semisolid Foods: Principles and Applications, Springer - Verlag (1999).

[10]. D. B. Braun and M. R. Rosen, Rheology Modifiers Handbook: Practical Use and Application, Noyes, Kansas, USA (2000).

[11]. G. B. Thurston, Rheological parameters for the viscosity, viscoelasticity, and thixotropy of blood, Biorheology, 16, pp. 149- 162, (1979).

[12]. K. K. Yeleswarapu, Evaluation of continuum models for characterizing the constitutive behavior of blood. Ph.D. Dissertation, University of Pittsburgh. Pennsylvania, USA (1996). 
[13]. K. R. Rajagopal and A. R. Srinivasa, A thermodynamic frame work for rate type fluid models, $J$. Non-Newtonian Fluid Mech. 88, 207-227 (2000).

[14]. M. Anand and K. R. Rajagopal, A shear-thinning viscoelastic fluid model for describing the flow of blood, Int. J. Cardiovasc. Med. Sci. 4, 59-68 (2004).

[15]. R. Fahraeus, T. Lindqvist, The viscosity of blood in narrow capillary tubes, Amer. J. Physiol., 96 562-568 (1931).

[16]. S. N. Majhi, L. Usha, Modeling the Fahraeus-Lindqvist effect through fluids of differential type, Int. J. Engng. Sci., 26, 503-508, (1988).

[17]. S. N. Majhi, V.R. Nair, Pulsatile flow of third grade fluids under body acceleration-Modeling blood flow, Int. J. Engng. Sci., 32, 839-846 (1994).

[18] M. Massoudi, T.X. Phuoc, Pulsatile Flow of a blood using second grade fluid model, Continn. Mech. Thermodyn., 16, 199-211 (2007).

[19]. M. Sajid, A. Zaman, N. Ali and A. M. Siddiqui, Pulsatile flow of blood in a vessel using an Oldroyd-B fluid, Int. J. Nonlinear. Sci. Num. Simul., 16, 197-206 (2015).

[20]. Md. A. Ikbal, S. Chakravarty and P. K. Mandal, Two-layered micropolar fluid flow through stenosed artery: Effect of peripheral layer thickness, Comput. Math. Appl., 230, 243-259, (2009).

[21]. D. S. Sankar, U. Lee, Two-fluid non-linear model for flow in catheterized blood vessels, Int. J NonLinear Mech., 43, 622 - 631 (2008).

[22]. M. Pires and A. Sequeira, Flows of generalized Oldroyd-B fluids in curved pipes, Prog. Nonlinear Differential Equations Applications, 80, 21-43 (2011).

[23]. N. Arada and A. Sequeira, Steady Flows of Shear-Dependent Oldroyd-B Fluids around an Obstacle, J. Math. Fluid Mech. 7, 451-483(2005).

[24]. G. Pontrelli, Pulsatile blood flow in a pipe, Computers \& Fluids. 27, 367-380, (1998).

[25]. G. Pontrelli, Blood flow through a circular pipe with an impulsive pressure gradient, Math. Mod. Meth. Appl. Sci., 10, 187-202 (2000).

[26]. H. B. H Mohamed and B. D. Reddy, Some properties of models for generalized Oldroyd-B fluids, Int. J. of Engg Sci., (2010).

[27]. J. G. Oldroyd., On the formulation of rheological equations of state, Proc. Royal Society of London, Series A, Mathematical and Physical Sciences, 200, pp. 523-541 (1950).

[28] A. C. Burton, Physiology and Biophysics of the Circulation, Introductory Text, Year Book Medical Publisher, Chicago,(1966).

[29] S. C. Ling, H. B. Atabek, A nonlinear analysis of pulsatile flow in arteries, J. Fluid Mech. 55, pp. 493-511 (1972). 
[30]. P. K. Mandal, An unsteady analysis of non-Newtonian blood through tapered arteries with a stenosis, Int. J. Nonlinear. Mech. 40, 151-164 (2005).

[31]. K. A. Hoffmann, S. T. Chiang, Computational Fluid Dynamics, Engineering Edition System, Wichita, Kansas USA, 1, pp. 67208-1078, (2000).

[32]. N. Ali, A. Zaman and M. Sajid, Unsteady blood flow through a tapered stenotic artery using Sisko model, Computers \& Fluids, 101, 42-49 (2014).

[33] R. Bhargava, S. Sharma, H.S. Takhar, T.A. Bég, O. Anwar Bég, T.K. Hung, Peristaltic pumping of micropolar fluid in porous channel-model for stenosed arteries, J. Biomechanics, 39, 1, S649-S650 (2006).

[34] O. Anwar Bég, R. Bhargava, Sughanda, S. Rawat, H. S. Takhar and T. A. Bég, Computational simulation of biomagnetic micropolar blood flow in porous (tissue) medium, $5^{\text {th }}$ World Congress in Biomechanics, Munich, Germany, July (2006).

[35] J.L. Curiel Sosa, O. Anwar Bég and J.M. Liebana Murillo, Finite element analysis of structural instability using a switching implicit-explicit technique, Int. J. Computational Methods Engineering Science and Mechanics, 14, 452-464 (2013).

[36] M.S. A. Hourai, A. Tounsi and O. Anwar Bég, Thermoelastic bending analysis of functionally graded material sandwich plates using a new higher order shear and normal deformation theory, Int. J. Mechanical Sciences, 76, 102-111 (2013).

[37] O. Anwar Bég, Tasveer A. Bég, R. Bhargava, S. Rawat and D. Tripathi, Finite element study of pulsatile magneto-hemodynamic non-Newtonian flow and drug diffusion in a porous medium channel, J. Mechanics Medicine Biology, 12 (4) 1250081.1 - 1250081.26 (2012).

[38] P. Nithiarasu, O. Hassan, K. Morgan, N.P. Weatherill, C. Fielder, H. Whittet, P. Ebden, and K.R. Lewis, Steady flow through a realistic human upper airway geometry, Int. J. Numerical Methods Fluids, 57:631-651 (2008).

[39] Reddy, J.N., An Introduction to the Finite Element Method, MacGraw-Hill, New York (1985).

[40] K.J. Bathe, Finite Element Procedures, Prentice-Hall, New Jersey, USA (1996). 